\title{
Rescuing the nephrologist's main central vein - placement of a right jugular vein catheter in a patient with thoracic central venous occlusion by use of the Surfacer ${ }^{\circledR}$ device
}

\author{
Nuno Afonso $0^{1,2}$, Luís Rodrigues ${ }^{1,2}$, Emanuel Ferreira ${ }^{1,2}$, Helena Pinto ${ }^{1,2}$, Rui Nogueira ${ }^{1}$, Gabriel Anacleto ${ }^{3}$, Manuel Fonseca ${ }^{3}$, Rui Alves $^{1,2}$ \\ ${ }^{1}$ Serviço de Nefrologia, Centro Hospitalar e Universitário de Coimbra \\ 2 Clínica Universitária de Nefrologia, Faculdade de Medicina da Universidade de Coimbra \\ ${ }^{3}$ Serviço de Cirurgia Vascular, Centro Hospitalar e Universitário de Coimbra
}

\begin{abstract}
Introduction: Central venous stenosis can be the main obstacle to the creation of a vascular access. The Surfacer ${ }^{\circledR}$ device was developed to solve the problem of complete occlusions of the right internal jugular vein, right brachiocephalic vein and superior vena cava, allowing for the placement of a vascular access in this venous axis. Case Report: We present the case of a 77-year-old patient on long-term hemodialysis with a long history of failed vascular accesses and of central vein stenosis, chronically dialyzed using a tunneled catheter placed through the left femoral vein. With use of the Surfacer ${ }^{\circledR}$ device it was possible to recreate, with an inside-out technique, a tract through the fibrous tissue of the completely occluded right brachiocephalic and right internal jugular veins, subsequently placing a tunneled catheter into the right atrium through this later vein. Conclusion: We report the first successful use in Portugal of a new vascular access device that allows us to rescue a previous unusable right central venous axis for the placement of a central venous catheter, thus avoiding the use of less advantageous options such as the femoral veins and preserving venous vascular capital.
\end{abstract}

Key words: Central Venous Occlusion, Central Veins, Surfacer ${ }^{\circledR}$

\section{INTRODUCTION}

Central venous stenosis (CVS) and occlusions (CVO) are common and frustrating problems in dialysis patients, regularly considered the "Achilles' heel" of vascular access (VA) ${ }^{1-2}$ Central veins include, in the cervical-thoracic region, the subclavian veins (SCV), the thoracic segment of the internal jugular veins (IJV), the brachiocephalic veins (BCV) and the superior vena cava (SVC), and, for the lower extremities, the iliac veins and inferior vena cava (IVC). ${ }^{2}$ Although the absolute incidence of CVS in dialysis patients is unknown, it is estimated that around $15-20 \%$ may develop symptomatic CVS. ${ }^{3}$

There is presently no completely satisfactory treatment for CVS/CVO and prevention is essential. Standard treatment of CVS is the performance of percutaneous angioplasty and, eventually, placement of a stent. Although usually immediately successful, it is associated with almost universal recurrence and need of repeated procedures. Hence, in the chronic kidney disease (CKD) population, avoidance of central venous catheters (CVC) and other vascular devices, such as peripherally inserted central lines (PIC lines) and cardiac implantable electronic devices (CIED) is crucial, since these are the key risk factors for the development of both these lesions. ${ }^{2,4-5}$

The development of complete central venous occlusions is particularly troublesome since its standard approach, "sharp recanalization", is not only difficult, potentially leading to life-threatening complications, but has also a significant failure rate.
The Surfacer ${ }^{\circledR}$ device was developed to provide a safe and reproducible way of reopening CVOs in the SVC, right BCV and right internal jugular veins (RIJV), allowing for the rescue of this central venous axis.

We describe the first use of the Surfacer ${ }^{\circledR}$ device by a Portuguese Interventional Nephrology Center and we share our experience with its use.

\section{CASE REPORT}

\section{Patient Vascular Access Background}

We present the case of a 77-year-old patient, on regular hemodialysis (HD) since January 2002 and with a long history of failed arterial-venous VAs. In June 2018, a venography performed for VA planning showed a complete occlusion of the right BCV and a small reduction of caliber in the left $B C V$, without the presence of collaterals. Upon failure of a left humeral-axillar AV graft secondary to occlusion of the left BCV, in June 2019, a tunneled CVC was placed in the left femoral vein (FV).

\section{Procedure Planning}

The patient was referred to our center for VA planning due to recurrent problems in achieving adequate blood flows during HD 


\section{Figure 1}

The Surfacer ${ }^{\circledR}$ device
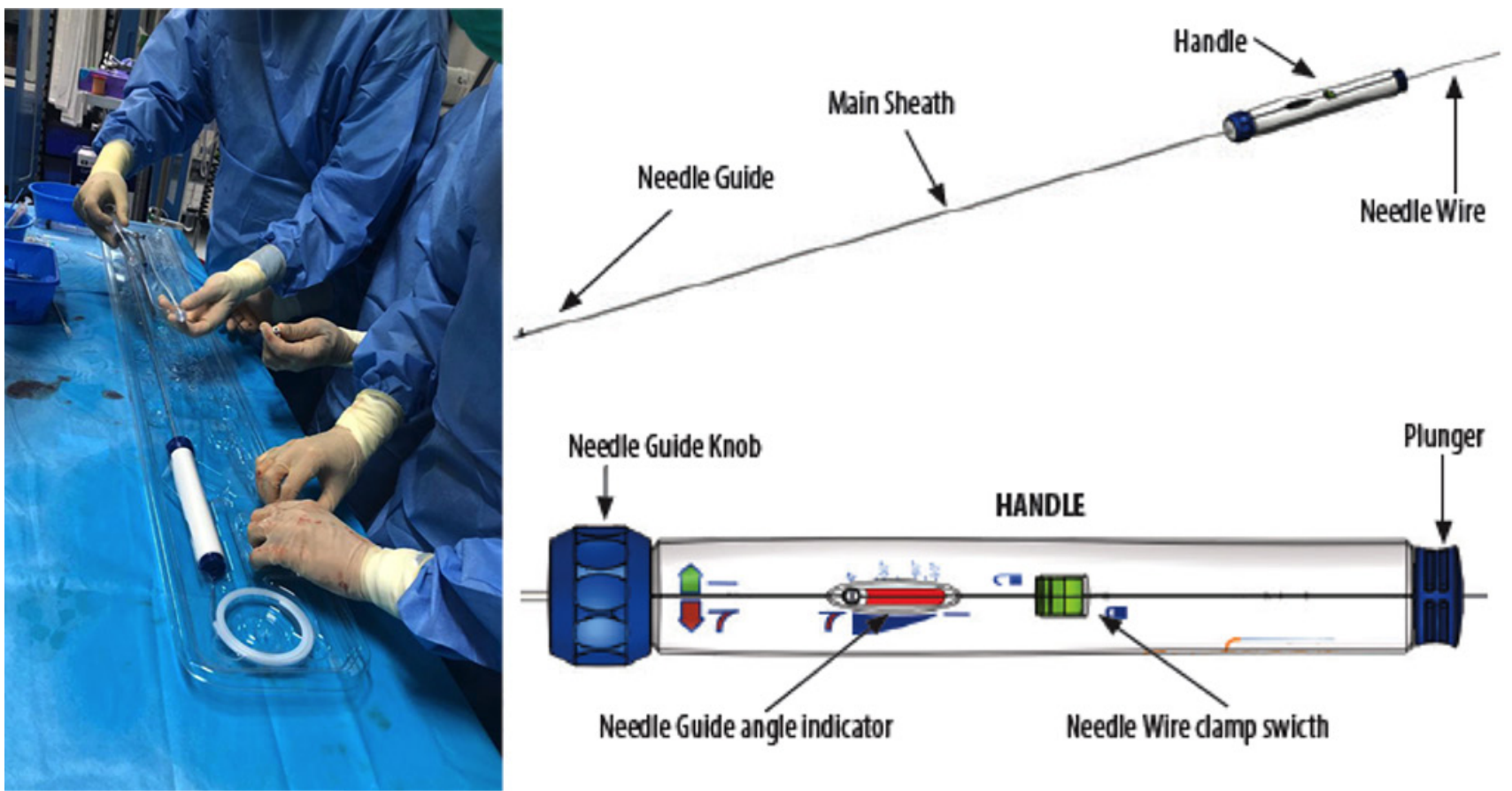

a) Main Sheath: it is the Surfacer ${ }^{\oplus}$ rigid steel rod, used to perform the blunt dissection of the occlusion, inside which runs the needle wire; b) Needle Guide: variable angle structure at the tip of the main sheath that determines the skin exit angle of the needle wire; c) Needle wire: stiff and sharpened guide-wire that will exit through the skin and will be used, subsequently to support the CVC insertion; e) Needle Guide Knob and Needle Guide angle indicator: handles that regulate the needle guide position; f) Plunger: handle that pumps the needle wire out.

sessions and low Kt/V. He had well-documented occlusion of both BCVs. With limited options available, we decided to try to reopen the right $\mathrm{BCV}$ and place a RIJV catheter, making use of the Surfacer ${ }^{\circledR}$ device.

The Surfacer ${ }^{\circledR}$ is a $95 \mathrm{~cm}, 7$ French (F), straight steel rod (Figure 1), designed to perform a blunt dissection of CVOs affecting the thoracic central veins: SVC, right BCV and intra-thoracic segment of the RIJV. It is based on the existence of a relatively straight line anatomy along the right femoral and iliac veins, IVC, right atrium and SVC axis, that allows for a simple navigation from the insertion point in the right FV up to the occluded thoracic central vein. The blunt dissection is performed while moving away from the major vascular structures of the mediastinum, thus minimizing the risks of the procedure.

Use of this device requires patency of the right femoral, iliac veins and IVC, and to have proper identification of the SVC. This last point is critical, since the right atrium appendage overlies the SVC in an AP projection and can be a source of confusion; failure to correctly identify the SVC stump and puncture of the atrium appendage can lead to hemopericardium and cardiac tamponade.

Proper pre-op evaluation should include an angio-CT or venography and vertebral column $\mathrm{x}$-ray (ensuring there are no significant deformities that may compromise a straight line navigation). Figure 2 presents the pre-op evaluation of our patient.
Additional pre-op checklist included informed consent signing and routine pre surgical evaluation; vascular surgery support was sought for the event of a major complication.

\section{Procedure and outcome}

The procedure was performed on the $23^{\text {rd }}$ of September 2019, on an in-patient basis, using the angiography room of the Cardiology Department. It was executed under conscious sedation with local anesthesia. Skin preparation was performed, both at the right inguinal and cervical/right anterior chest areas. Antibiotic prophylaxis was performed with cephazolin and ceftazidime.

Access to the right FV was secured through placement of an $8 \mathrm{~F}$ introducer. A 0,035" guide-wire was passed up to the SVC and a $12 F, 45 \mathrm{~cm}$ long vascular sheath was introduced up to the level of the occlusion in the right BCV, Figure $3 a$ (a Flexor ${ }^{\circledR}$ Check-Flo ${ }^{\circledR}$ Introducer by Cook Medical was used). Subsequently, the Surfacer ${ }^{\circledR}$ workstation sheath was introduced telescopically inside the first one (Figure $3 b$ ). After successfully securing access to the CVO, the exit target (simply a small ring that acts as a radiopaque marker) was placed on the desired exit point in the supraclavicular exit area (roughly on the same location where one would puncture to access the RIJV).

After these preliminary steps, the Surfacer ${ }^{\circledR}$ device's main sheath was introduced through the RFV, inside both the previous sheaths, and gently pushed up to the occlusion level (Figure 3c). 


\section{Figure 2}

Pre-op evaluation
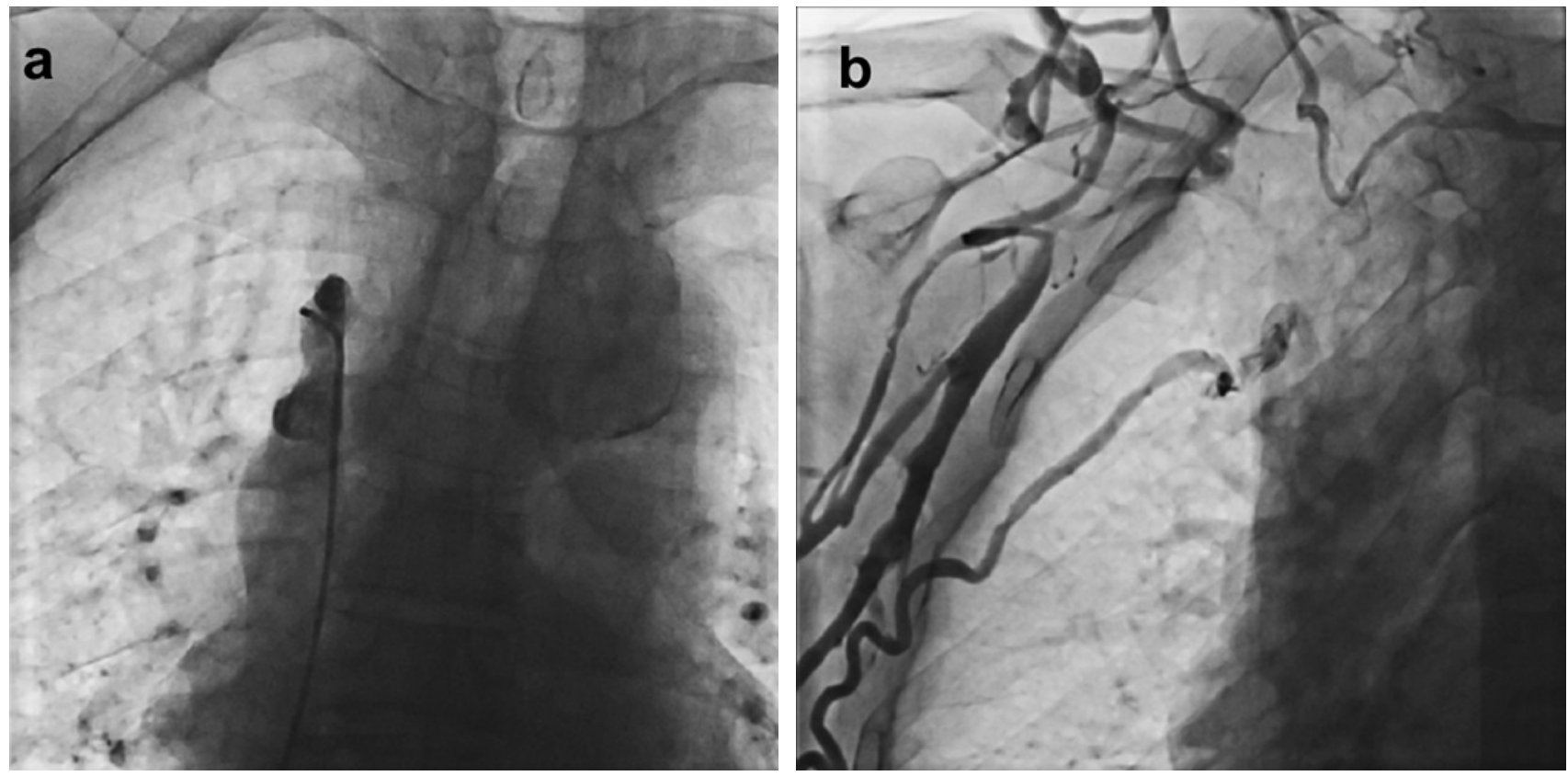

a) location of the CVO of the right BCV after insertion of a diagnostic catheter by way of the right femoral vein - notice the proper patency of the SVC; b) image of the central veins by way of right upper arm venography.

At this point, under fluoroscopic guidance, blunt dissection of the occluded path was achieved by gently pushing up the device until reaching the cervical area immediately above the right clavicle (Figure 3d).

Alignment of the Surfacer ${ }^{\circledR}$ tip with the skin marker was achieved both by rotating the device laterally (Figure 3e) and rotating the image intensifier cranially. The rotation angle of the image intensifier was recorded at around 40 , and translated to the Surfacer ${ }^{\circledR}$ handle, thus determining the proper angle of the Surfacer ${ }^{\circledR}$ needle guide.

Having achieved these steps, a sharpened and stiff guide-wire was pumped out of the device (Figure $3 \mathrm{f}$ ); it exited through the skin at the level of the radiopaque steel marker (Figure 3g). At this point we had through and through access to the venous circulation and a successful outcome for the procedure was virtually guaranteed.

A split-sheath from a tunneled CVC was next inserted at the cervical level using the previously mentioned guide-wire (Figure $3 \mathrm{~h}$ ). To do this, the wire was inserted in the sheath and clamped at the exit point; the wire was then pulled at the femoral level, thus pulling the split-sheath with it. After this a tunneled CVC was placed using the standard technic (a Medcomp ${ }^{\circledR}$ Ash-Split catheter with $24 \mathrm{~cm}$ total length was used); it was perfectly placed with both tips inside the right atrium and it offered no resistance to blood flow (Figure 3i).

The femoral introducer placed in the RFV and the tunneled CVC in the LFV were both removed at the end of the procedure. Total procedure time was approximately one hour and total fluoroscopy time was 4.9 minutes with an effective x-ray dosage of 258 UGy.
The new CVC performed well during the dialysis session and the patient was discharged after a 24-hour surveillance period. The initial plan was to, subsequently, replace the $\mathrm{CVC}$ with a $\mathrm{HeRO}^{\circledR}$ device; the patient, however, refused further procedures. The CVC has been in place for around 9 months and has been performing well without infectious events.

\section{DISCUSSION}

The development of CVS/CVO is a serious problem for dialysis patients, utterly compromising the options for creation of hemodialysis VA. The incidence of complete CVO in dialysis patients is unknown. Our personal perception is that the problem is significantly underestimated, since these cases are usually unreported and, commonly, the physician simply moves on to the next venous vascular site available.

The recanalization of a CVO is a potentially complicated procedure, often requiring performance of a "sharp recanalization" of the vein. This is not a standardized procedure, requiring a very experienced operator, and is associated with significant risks. ${ }^{2,6} \mathrm{~A}$ recent series of 39 patients reported a $95 \%$ success rate in the placement of a dialysis access through a CVO with use of sharp recanalization. ${ }^{7}$ However, even in this experienced center, two patients (5\%) developed pericardial hemorrhages. ${ }^{7}$

In our own center, despite the absence of serious complications, in a review of CVSs approached from 2009-2014, 5 out of 31 procedures (16\%) failed due to inability to overcome a CVO. ${ }^{8}$ 


\section{Figure 3}

The procedure
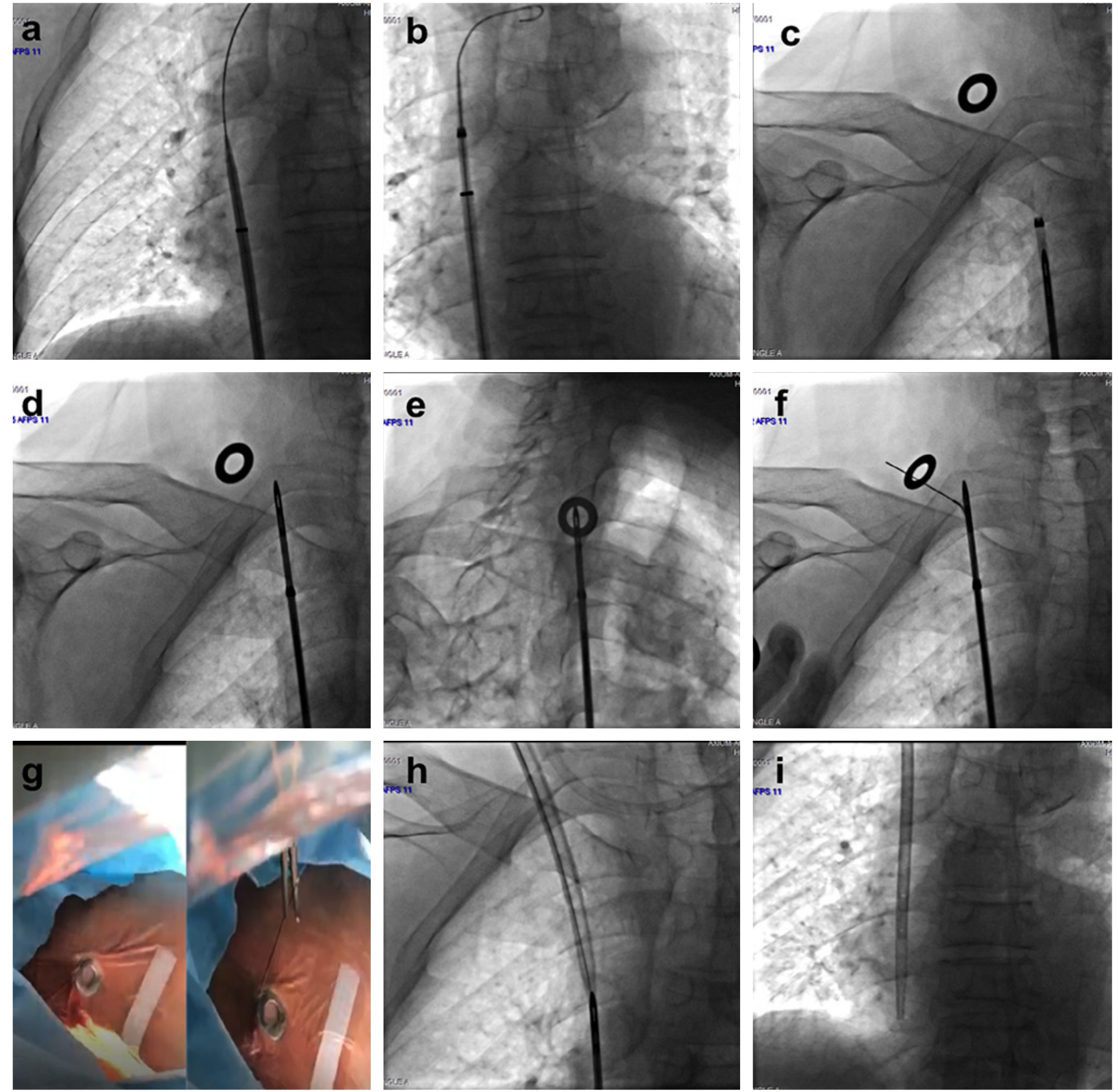

a) inserting the outer $12 \mathrm{~F}$ vascular sheath; $\boldsymbol{b}$ ) insertion of the Surfacer ${ }^{\otimes}$ workstation sheath; $\boldsymbol{c}$ ) pushing the Surfacer ${ }^{\otimes}$ main sheath up to the occlusion location inside the previous sheaths (notice the radiopaque ring marker pointing the exit site area at the cervical level); d) performing the blunt dissection of the occluded venous segment up to the cervical area above the right clavicle; e) aligning the opening on the Surfacer ${ }^{\otimes}$ tip with the radiopaque ring target; f) pumping the needle wire out through the skin - fluoroscopic view; g) pumping the needle wire out through the skin - view from the cervical area, immediately before and after the wire punching out through the skin; $\boldsymbol{h}$ ) inserting the catheter split-sheath by pulling the needle wire and the Surfacer main-sheath at the femoral level and not by pushing the CVC sheath in; i) proper placement of the CVC with both tips inside the right atrium.

The problem of CVO is not exclusive to Nephrology and CKD patients. With the ever-expanding indications for CIED and the frequent need to add or replace existing leads, cardiologists are also increasingly facing the problem of CVOs. ${ }^{9-10}$
Indeed, the original concept of inside-out reopening of CVOs was developed as a new technique of lead placement for patients with this type of venous pathology. ${ }^{11}$ It makes use of the consistent anatomical relationships of the thoracic vascular structures and the fact 
that the thoracic central veins are bounded anteriorly only by soft tissues, clavicle and skin. ${ }^{11}$ Therefore, a needle directed anteriorly from a central vein will not encounter arteries, nerves or pleura that lie posterior and lateral to the central veins. ${ }^{11}$ Standardization of this technique led to the creation of the Surfacer ${ }^{\circledR}$ device; it was approved for clinical use in Europe (CE Marketed) in July 2016.

A recently published study accessed the eligibility and success rate of this "inside-out" approach for the placement of right-sided CVCs in dialysis patients with complete CVO. ${ }^{12}$ In total, 36 patients were included, of which 30 (83\%) had CVOs types 3 and 4 (meaning bilateral occlusion of both BCVs, and perceived to be running out of dialysis accesses). The success rate was $97 \%$ (38 out of 39 procedures were successful), with proper placement in the right IJV of a tunneled dialysis CVC. Also noteworthy, $72 \%$ of the procedures were executed by interventional nephrologists and there were no complications with the procedures. The average time of the entire procedure was 43 minutes, with an average total fluoroscopy time of only 6 minutes. ${ }^{12}$ Similar successful results are also reported in recently published small case series. ${ }^{13-14}$

Our case is, to the best of our knowledge, the first experience with the Surfacer ${ }^{\circledR}$ device and the "inside-out" technique reported by a Portuguese Interventional Nephrology Center and was successfully accomplished in a 77-year-old patient. With this technique we were able to successfully reopen a completely occluded main central venous axis and place a tunneled right IJV catheter in a patient who was running out of VA options and dependent on a femoral CVC, with associated poor dialysis efficiency, increased risk of infections and lesser quality of life. The procedure was remarkably straightforward, with a total procedure time of around one hour. The exceedingly low total fluoroscopy time, under 5 minutes, was also remarkable and in line with the average time reported by larger series.

Contributing to this successful outcome was the proper pre-op evaluation, ensuring suitable mapping of the occluded venous segments and that there would be no surprise abnormal anatomical findings during the procedure. We emphasize the extra care taken to avoid complications during the Surfacer ${ }^{\circledR}$ insertion, by previously inserting an extra vascular sheath; this initial step, although not mandatory, gives an extra insurance that the device will proceed, with no deviations, along the proper vascular route.

The main limitation of this device is that, by its configuration, it is limited to the right central venous axis and requires proper patency of the right femoral, iliac veins and IVC. It is also costly (around $3000 €$ ) and requires, for safety reasons, a vascular access center with prompt access to thoracic/cardiac and vascular surgery. Although difficult to compare, these costs must be balanced against the previously mentioned costs of under dialysis and increased risk of infections and, eventually, hospitalization and need to replace the VA.

In summary, this technique allowed us to solve a challenging case of dialysis vascular access exhaustion by recycling a previously unusable vascular axis. In allowing a consistent way to solve the problem of complete venous occlusions in the right IJV, BCV and SVC axis (the preferred venous axis for CVC emplacement in dialysis patients), it represents a significant tool for the nephrology community, allowing for the continued use of this venous axis, sparing other vascular territories and increasing the vascular access options in dialysis patients.

Disclosure of potential conflicts of interest: none declared

\section{References}

1. Altman S. A practical approach for dagnosis and treatment of central venous stenosis and occlusion. Semin Vasc Surg 2007;20:189-194.

2. Horikawa M, Quencer K. Central venous interventions. Tech Vasc Interventional Rad 2017;20:48$-57$.

3. Ibeas J, Roca-Tey R, Vallespín J, et al. Guía Clínica Española del Acceso Vascular para Hemodiálisis. Nefrologia. 2017;37 Suppl 1:1-191.

4. Modabber M, Kundu S. Central venous disease in hemodialysis patients: an update. Cardiovasc Intervent Radiol 2013;36:898-903

5. Thoracic central vein occlusion associated with hemodialysis access. Available at Uptodate. Accessed May 82020.

6. Worley SJ, Gohn DC, Pulliam RW, et al. Subclavian venoplasty by the implanting physicians in 373 patients over 11 years. Heart Rhythm 2011;8(4):526-533.

7. Cohen El, Beck C, Garcia J, et al. Success rate and complications of sharp recanalization for treatment of central venous occlusions. Cardiovasc Intervent Radiol. 2018;41(1):73-79.

8. Pinto $\mathrm{H}$, Ferreira $\mathrm{E}$, Afonso $\mathrm{N}$, et al. Interventional nephrology - five years dealing with central stenosis: immediate and long-term results. Port J Nephrol Hypert 2018;32(3):245-249.

9. Marcial J, Worley S. Venous system interventions for device implantation. Card Electrophysiol Clin 2018;10:163-177.

10. Da Costa SS, Scalabrini Neto A, Costa R, et al. Incidence and risk factors of upper extremity deep vein lesions after permanent transvenous pacemaker implant: a 6 month follow-up prospective study. Pacing Clin Electrophysiol 2002;25(9):1301-1306.

11. Elayi C, Allen C, Leung S, et al. Inside-out access: a new method of lead placement for patients with central venous occlusions. Heart Rhythm 2011;8(6):851-7.

12. Schwaighofer RR, Matoussevitch V, Winnicki W, et al. A novel inside-out access approach for hemodialysis catheter placement in patients with thoracic central venous occlusion. AJKD 2020;75(4):468-470.

13. Hentschel DM, Minarsch L, Vega F, Ebner A. The Surfacer ${ }^{\circledast}$ Inside-Out ${ }^{\oplus}$ Access System for right-sided catheter placement in dialysis patients with thoracic venous obstruction. J Vasc Access. 2020;21(4):411-418.

14. Quek LH, Tan TS, TAN GW, et al. Salvage of exhausted neck access using a novel inside-out device in dialysis-dependent patients. Hemodial Int. 2019;23(4):E111-E114.

\section{Correspondence to:}

Nuno Afonso

Serviço de Nefrologia - Centro Hospitalar e Universitário de Coimbra

Praceta Mota Pinto - 3000 Coimbra

Email: nunomacoliveira@gmail.com 